\title{
Post-compression of high average power picosecond pulses for few cycle generation and FEL pump-probe experiments
}

\author{
Anne-Lise Viotti ${ }^{1,2}$, Skirmantas Alisauskas ${ }^{1}$, Prannay Balla ${ }^{1,3}$, Ammar Bin Wahid ${ }^{1}$, Ivan Sytcevich ${ }^{2}$, Chen \\ Guo $^{2}$, Laura Silletti ${ }^{1}$, Andrea Cartella ${ }^{4}$, Hamed Tavakol ${ }^{1}$, Uwe Grosse-Wortmann ${ }^{1}$, Arthur Schönberg ${ }^{1,3}$, \\ Marcus Seidel $^{1}$, Bastian Manschwetus ${ }^{1}$, Tino Lang ${ }^{1}$, Andrea Trabattoni ${ }^{1}$, Francesca Calegari ${ }^{14,5}$, Arnaud \\ Couairon ${ }^{6}$, Anne L'Huillier ${ }^{2}$, Cord L. Arnold ${ }^{2}$, Ingmar Hartl' ${ }^{1}$ and Christoph M. Heyl ${ }^{1,3}$
}

${ }^{1}$ Deutsches Elektronen-Synchrotron DESY, Notkestraße 85, 22607 Hamburg, Germany

${ }^{2}$ Department of Physics, Lund University, P.O Box 118, SE-221 00 Lund, Sweden ${ }^{3}$ Helmholtz-Institute Jena, Fröbelstieg 3, 07743 Jena, Germany

${ }^{4}$ The Hamburg Centre for Ultrafast Imaging, Universität Hamburg, 149 Luruper Chaussee, Hamburg 22761, Germany ${ }^{5}$ Institut für Experimentalphysik, Universität Hamburg, Luruper Chaussee 149, 22761 Hamburg, Germany

${ }^{6}$ Centre de Physique Théorique, CNRS, Ecole Polytechnique, Institut Polytechnique de Paris, F-91128 Palaiseau, France

Ultrafast Ytterbium-based $(\mathrm{Yb})$ amplifiers can be power-scaled into the kilowatt $(\mathrm{kW})$ regime, offering great potential for applications where repetition rate scaling of ultrafast lasers beyond the average power limits of Ti:sapphire technology is needed. Examples are future laser-driven accelerators and pump-probe experiments at superconducting X-ray free electron lasers (FEL). However, the gain bandwidth of $\mathrm{Yb}$ media limits the output pulse durations of such amplifiers to a few $100 \mathrm{~s}$ of fs to $1 \mathrm{ps}$. External spectral broadening concepts can be employed for pulse post-compression, offering the possibility to combine ultrashort pulse durations, high repetition rates and milli-joule $(\mathrm{mJ})$ level pulse energies. In particular, a recently developed post-compression scheme based on spectral broadening in gas-filled multi-pass cells (MPC), shows good prospects for peak- and average-power scaling [1].

Here, we demonstrate a compression factor of about 40 in a single pulse compression stage, compressing $1.2 \mathrm{ps,}$ $2 \mathrm{~mJ}$ pulses centered at $1030 \mathrm{~nm}$ to $32 \mathrm{fs}$ at $200 \mathrm{~W}$ in-burst average power. A second MPC stage is used to reach pulse durations down to $13 \mathrm{fs}$, thus opening a route towards high average power few-cycle sources driven by commercial high-power ps lasers [2]. The experimental setup is shown in Fig. 1 (a). It consists of two compression stages containing mode-matching units, gas-filled MPCs and dispersion compensating mirrors (DCMs). The cells are operated close to the stability edge, at lengths close to $2 \mathrm{~m}$, with 44 passes for the first cell and 12 passes for the second cell. The compressed output pulses are characterized by SHG-FROG and dispersion scan, respectively. The results are shown in Fig.1 (b).
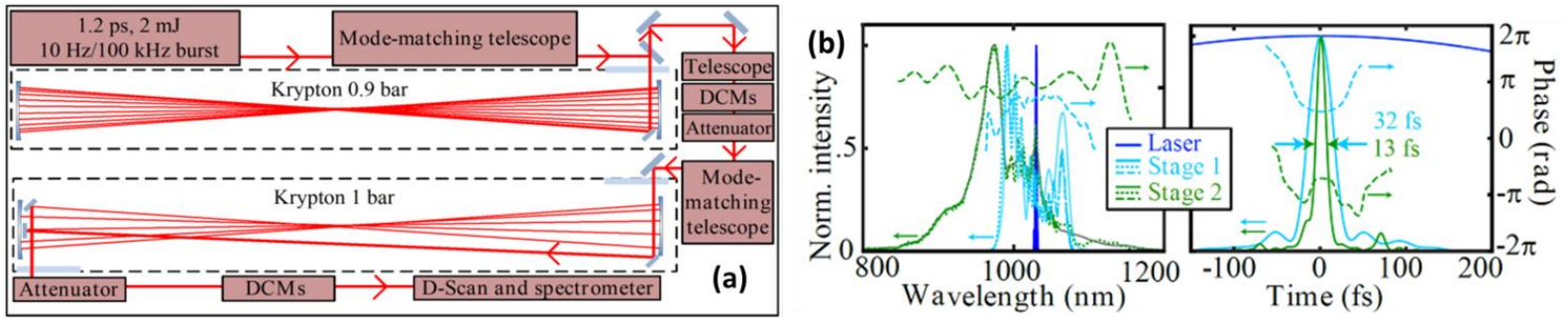

Fig. 1 (a) Experimental setup consisting of two gas-filled MPCs, mode-matching optics and DCM compressors. (b) Reconstructed spectral and temporal intensity (solid) and phase (dashed) profiles together with the corresponding measured spectra after both MPCs (dotted) and at the laser output (dark blue). SHG-FROG data (stage 1) are shown in light blue and dispersion scan data (stage 2) are shown in green.

The throughput of the first MPC is $85 \%$ (80\% including the DCM compressor). The throughput of the second MPC is $46 \%$, limited mainly by losses at the MPC silver mirrors, yielding $0.37 \mathrm{~mJ}$ pulses at the output.

These results are obtained employing a commercial Yb;YAG Innoslab amplifier system, which is currently used as pump for the OPCPA pump-probe laser system at the FLASH FEL facility. With a relatively simple addon, the here demonstrated post-compression unit can complement the parameter range of the FLASH pump-probe laser system available for laser-FEL pump-probe spectroscopy [3]. In particular, long-term stability measurements are carried out with a single MPC in preparation for first FEL user experiments. Over $36 \mathrm{~h}$, MPC output pulse energy fluctuations are below 4\% RMS (2\% at the laser output) for compressed output pulses of about 55 fs duration and a standard deviation of $2.8 \mathrm{fs}$. These results, together with a well-preserved beam quality through the MPC ( $\mathrm{M}^{2}$ of $1.3 \times 1.2$ vs. $\mathrm{M}^{2}$ of $1.2 \times 1.1$ for the laser output), show that such a post-compression system is suitable for various ultrafast measurements including applications which demand long-term stable operation.

\section{References}

[1] J. Schulte et al., "Nonlinear pulse compression in a multi-pass cell", Opt. Lett. 41, 4511 (2016).

[2] P. Balla et al., "Postcompression of picosecond pulses into the few-cycle regime“", Opt. Lett. 45, 2572 (2020).

[3] J. Rossbach et al., "10 years of pioneering X-ray science at the Free-Electron Laser FLASH at DESY," Phys. Rep. 808, 1 (2019). 